\title{
Design of a machine vision applied to educational board game
}

\author{
Carlos Calderon-Cordova ${ }^{1+}$, María Guajala-Michay ${ }^{2}$, Rodrigo Barba-Guaman ${ }^{1}$ and Pablo \\ Quezada-Sarmiento ${ }^{1}$ \\ ${ }^{1}$ Department of Computer Science and Electronics, Technical University of Loja, Loja, Ecuador \\ ${ }^{2}$ Department of Architecture and Arts, Technical University of Loja, Loja, Ecuador
}

\begin{abstract}
This paper proposes the architecture and the procedure for the design of a new computer vision based educational board game, which is an automated tutor oriented to special education institutions. For practical demonstration of the proposed system, we applied machine vision to educational board game focused on Braille math operations two-dimensional. The performance evaluation of machine vision based on cross-correlation algorithm obtained a success rate of $94.6 \%$. The result of this work will serve to implement a tool low-cost, fast and non-intrusive to automate tutoring of educational board games in special education institutions.
\end{abstract}

Keywords: Automated tutor, educational board game, machine vision, visually impaired children

\section{Introduction}

In Ecuador, according to the latest population census conducted in November 2010, there are 186.117 visually impaired people, and 17.081 is population less than 12 years, that is to say there are 1.2 visually impairment children (VIC) per 1000 inhabitants [1]. Nevertheless most special education institutions lack educational material aimed to VIC, on this basis in [2] it is proposed to generate educational material to enhance learning of mathematics in blind children, as a result of the project a wide set of educational board games applied to the main topics of basic education in mathematics was obtained, however these games need a teacher to monitor and feedback the operations performed by the VIC, this is a problem when these games are applied in classrooms of 10 or more VIC.

Referring to the problem identified, the scientific community has created some assistive technologies in the learning processes of visually impaired children (VIC). For example in [3] is developed an Audio Video Describer using narration to visualize movie films; in [4] is presented an embedded application (Portáctil) to aid the visually impaired that allows reading and writing printed or digitized documents; in [5] is exposed the design, development and usability of two based on audio virtual environments (AudioMath and Theo \& Seth) that increase mathematics learning in VIC.

Based on the literature research, our contribution is the design of an automated tutor applied to educational board games for VIC, focused on math topics. The proposed system is a new application of machine vision, which allows automatically inspect the area of the game based on processing of the real-time acquired image, additionally, the feedback messages to the child are executed by voice signals. For the development of this project, first we analysed the characteristics of the educational board game (section 2). Then we defined the architecture of the machine vision and the procedure to be executed (section 3), section 4 describes each of the phases of defined procedure, in section 5 we present the experimental results at laboratory, finally, the main conclusions of the research project are summarized in section 6 .

\footnotetext{
Corresponding author. Tel.: + 593-73701444 ext. 2516

E-mail address: cacalderon@utpl.edu.ec.
} 


\section{Description of Educational Board Game}

The educational board game (EBG) is a tool to teach mathematical operations to visually impaired children. The EBG consists of two elements, the game board and the set of 56 tabs. With respect to the game board, the first row is a reference for the child, because it indicates the positions of the units, tens, hundreds, thousands and the number sign. The second and third row, have the boxes to enter the first and the second number up to 4 digits, also in the third row it is the box to enter the symbol of the mathematical operation to be performed. Finally, the fourth row consists of the boxes to enter the result of the operation between the numbers entered; this result can be up to 5 digits. With respect to gaming tabs, each have their respective symbol $(1,2,3,4,5,6,7,8,9,0,+,-, \mathrm{x}$, \#) supplemented with the corresponding Braille code and a distinctive color, this last feature is helpful for children who have partial visual impairment. The Braille code developed embossed is used to identify the symbol by touch [2]. Fig. 1a shows the elements of the educational board game, and Fig. $1 \mathrm{~b}$ shows an example of using the board and tabs of the educational board game focused on Braille math operations two-dimensional.

In this educational board game, is necessary a teacher who monitors and feedback the activities of the VIC, such as the child wants to know if entered correctly the numbers, or also the child needs to identify whether the entered result is correct or incorrect. Knowing this type of educational games is applicable to special educational institutions; potential problems are foreseen if these games are used in classrooms of 10 or more VIC. Based on the above, we propose to design an automated tutor based on machine vision, applied to educational board games for VIC, focused on math topics of basic education.

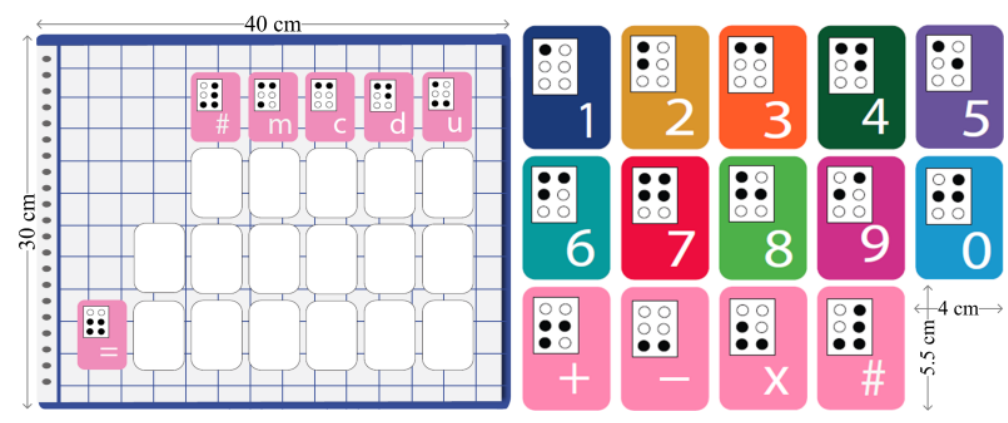

a)

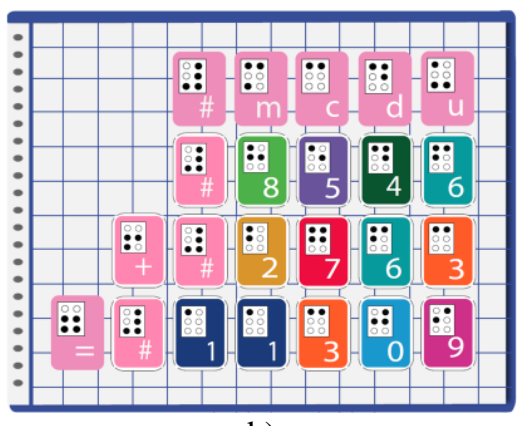

b)

Fig. 1: a) Elements of the educational board game. b) Example of using the board and tabs of the educational board game focused on math operations.

\section{Machine Vision: Architecture}

The central processing element is the personal computer that uses three peripheral devices: digital camera, speakers, and a keypad. The monitor software that controls the educational board game was called Axcess, this tool was developed in LabVIEW 2014 programming language from National Instruments, and it uses the functions of Vision Development Module (VDM) which is a library of commands to develop image processing applications. The LabVIEW and VDM software is widely used for the development of machine vision systems, for example in [6], [7].

Regarding the function of peripheral devices; the Genius FaceCam 1020 commercial camera captures the image in real time with a maximum acquisition rate of $30 \mathrm{fps}$ (frames per second) with a resolution of $1280 \times 720$ pixels. The speakers are the sound interface device from the computer to the VIC, by means of this device the computer communicates to the child about the state of educational game, by playing error and information messages which are generated automatically by the Axcess software.

Finally, the keypad is the touch interface between the VIC and the computer, it has four buttons marked with the letters $\mathrm{a}, \mathrm{b}, \mathrm{c}$, and $\mathrm{d}$; the function of the $\mathrm{a}$ and $\mathrm{b}$ buttons is to recognize the first and the second number respectively; c button function is to recognize the mathematical operation and check the result entered; and d button function is to recognize and check all numbers entered on the game board.

\section{Machine Vision: Procedure}

The Axcess processing software executes a sequence of steps, from the acquisition of the image in real- 
time until to check the result of EBG. The procedure (Fig. 2) which performs Axcess, is outlined: Read the image acquisition device. Segment the image of the game board. Extract and learn the symbolic patterns of the tabs. Determine regions of belonging to the boxes. Identify the symbolic patterns. Determine the symbolic matrix, and, Locate errors and check the result.

\section{Experimental Results}

The experimental setup for lab test is shown in Fig. 3. Boxes 1, and 2 show the educational board game and the set of tabs; box 3 shows the commercial camera Genius FaceCam 1020 with video resolution of $1280 \times 720$ pixels; box 4 shows the computer used for performing the procedure of machine vision, the model we used is Hewlett Packard Pavilion 14 Core i7; finally, box 5 shows the front panel of the Axcess software.

Two tests were performed: 1) Test of performance of the machine vision based on cross-correlation algorithm, and 2) Test of the algorithm that locates errors on the educational board game. Tests of performance of the machine vision we conducted in laboratory. We performed 25 experiments for the 14 tab types; for different positions and quantities; the experiments were distributed as follows: Ten experiments were performed with the game board horizontally oriented with respect to the camera (Ehor). Five experiments were performed with the game board with an inclination angle of from $5^{\circ}$ to $15^{\circ}$ in the direction of clockwise $(E c w)$. Five experiments were performed with the game board with an inclination angle from $5^{\circ}$ to $15^{\circ}$ counterclockwise to clockwise $(E c c w)$. Finally five experiments with the variable distance between the game board and the camera (Edvar); variation of distance is from $45 \mathrm{~cm}$ to $55 \mathrm{~cm}$. The success rates $(S r)$ in the experiments are shown in Table I and in Fig. 4. Then we performed the test of the algorithm that locates errors on the educational board game, the Fig. 5 shows the results of the $\boldsymbol{D}$ matrix and of the error messages for some specific cases of the game board.

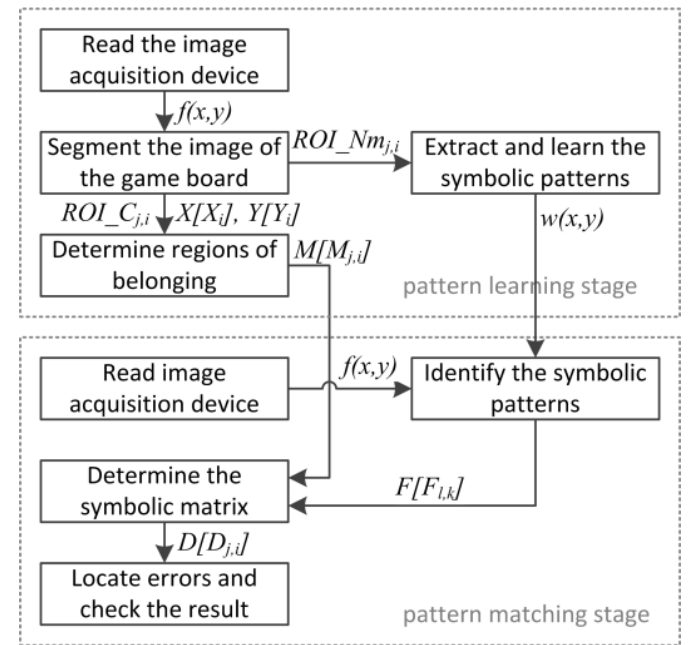

Fig. 2: Procedure Flowchart performed in the Axcess processing software.

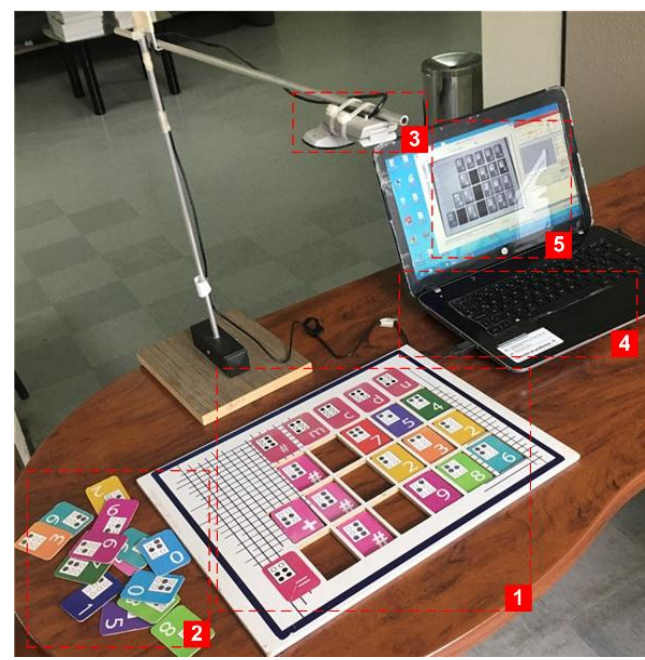

Fig. 3: Experimental setup for machine vision system.

\section{Conclusions}

We design, and tested an automated tutor applied to educational board games for visually impaired children; focused on math topics. Experimental tests of the architecture, and the procedure proposed, is demonstrated by applying of the system to the educational board game for Braille mathematical operations two-dimensional. The developed system is a new application of machine vision; it consists of a computer used as a central processing element and three peripheral devices: digital camera, speakers, and a keypad. Two tests were performed: 1) Test of performance of the machine vision based on cross-correlation algorithm, and 2) Test of the algorithm that locates errors on the educational board game. As a result of 1), the average success rate for the Ehor, Ecw, Eccw, and Edvar experiments; is equal to 94.6\%. Finally as a result of 2), the algorithm locates $100 \%$ of possible errors. These results indicate the high potential of the new application proposed of the vision machines; as a means for creating automated tutors applied to educational board games for visually impaired children. 
TABLE I: SUCCESS RATES IN THE EXPERIMENTS

\begin{tabular}{llllll}
\hline \hline Symbol & Ehor_Sr & Ecw_Sr & Eccw_Sr & Edvar_Sr & Avg_Sr \\
\hline 1 & 100 & 100 & 100 & 100 & 100 \\
2 & 100 & 100 & 100 & 100 & 100 \\
3 & 80 & 80 & 100 & 80 & 85 \\
4 & 100 & 100 & 100 & 100 & 100 \\
5 & 100 & 100 & 100 & 100 & 100 \\
6 & 100 & 80 & 80 & 100 & 90 \\
7 & 100 & 100 & 100 & 100 & 100 \\
8 & 90 & 80 & 100 & 80 & 87.5 \\
9 & 100 & 80 & 80 & 100 & 90 \\
0 & 100 & 100 & 80 & 100 & 95 \\
+ & 100 & 100 & 100 & 100 & 100 \\
- & 90 & 80 & 60 & 80 & 77.5 \\
$x$ & 100 & 100 & 100 & 100 & 100 \\
$\#$ & 100 & 100 & 100 & 100 & 100 \\
\hline \hline
\end{tabular}

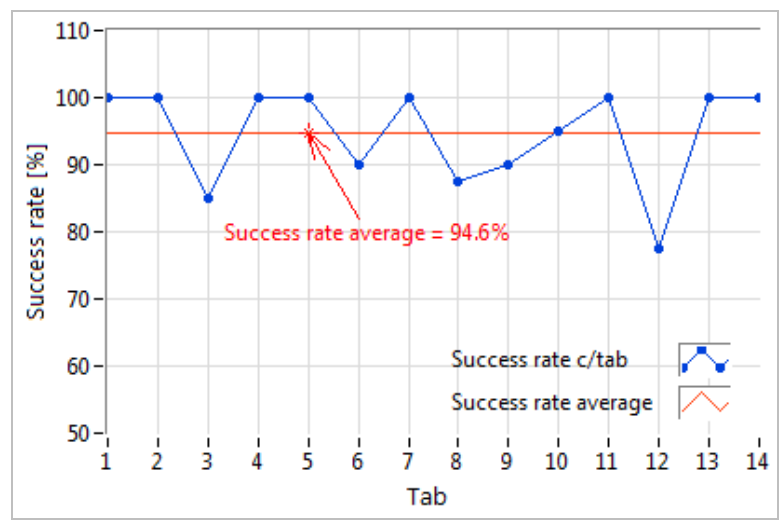

Fig. 4: Success rate average for each gaming tab.
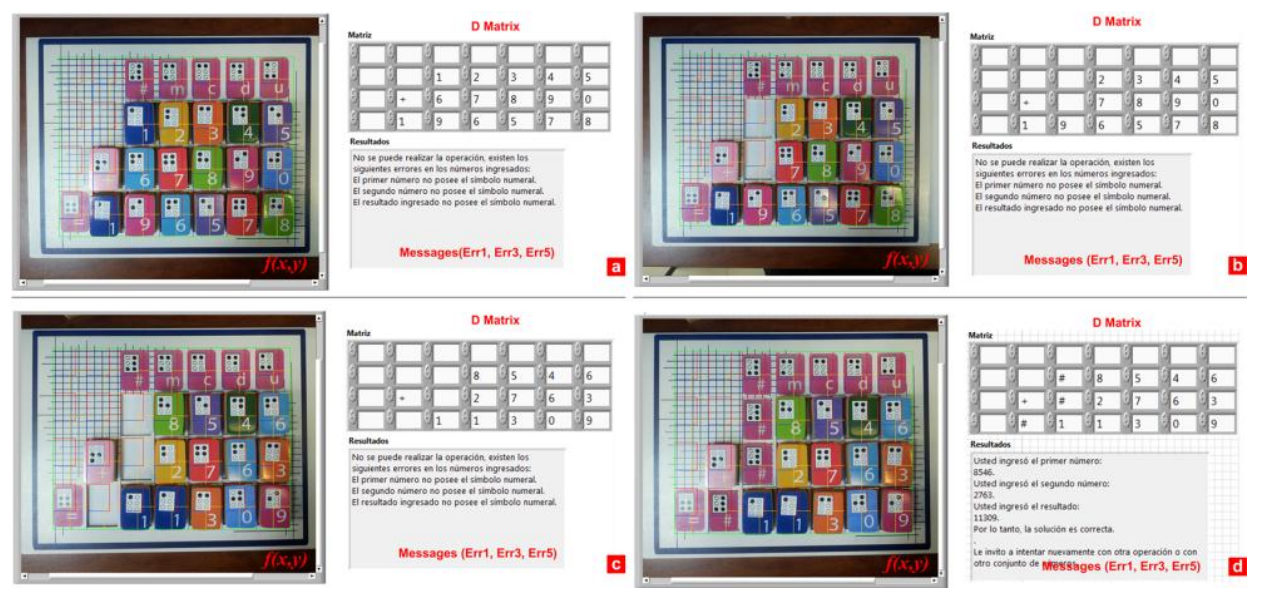

Fig. 5: Results of the $D$ matrix and of the error messages for some specific cases of the game board.

\section{References}

[1] Population by disability status, by province, county, parish, and registration area / Población por condición de discapacidad, según provincia, cantón, parroquia y área de empadronamiento (CPV-2010), Instituto Nacional de Estadística y Censos, Quito, Ecuador, November 2011.

[2] M. Guajala, "Generation of teaching materials to enhance learning of mathematics in blind children of the Loja city / Generación de Material Didáctico para mejorar el aprendizaje de las Matemáticas en los niños ciegos de la ciudad de Loja," M. S. Thesis, Dept. Arts and Design, UNAM, México, D.F., 2013.

[3] S. Firdus, W. F. W. Ahmad and J. B. Janier, "Development of Audio Video Describer using narration to visualize movie film for blind and visually impaired children," Computer \& Information Science (ICCIS), 2012 International Conference on, Kuala Lumpeu, 2012, pp. 1068-1072.

[4] L. R. R. Coutinho, A. M. Girão, J. B. B. Frota and E. T. Silva Jr., "Device to Assist the Visually Impaired in Reading Printed or Scanned Documents," Computing System Engineering (SBESC), 2012 Brazilian Symposium on, Natal, 2012, pp. 25-30.

[5] J. Sánchez and H. Flores, "Training blind children to develop mathematics skills through audio," CyberPsychology \& Behavior, 2005, vol. 8, no. 4, pp. 354-355.

[6] Haitao Zhu, Jianming Xu, Defeng He, Kexin Xing and Zhanglei Chen, "Design and implementation of the moving workpiece sorting system based on LabVIEW," Control and Decision Conference (2014 CCDC), The 26th Chinese, Changsha, 2014, pp. 5034-5038.

[7] P. Hryniewicz, W. Banaś, A. Gwiazda, K. Foit, A. Sękala and G. Kost, "Technological process supervising using vision systems cooperating with the LabVIEW vision builder," IOP Conference Series: Materials Science and Engineering, vol. 95, no. 1, pp. 1-6, 2015. 